Jhohan Chavez*, Valter Böhm, Tatiana I. Becker, Simon Gast, Igor Zeidis and Klaus Zimmermann

\title{
Actuators based on a controlled particle-matrix interaction in magnetic hybrid materials for applications in locomotion and manipulation systems
}

\begin{abstract}
The paper deals with the investigation of magneto-sensitive elastomers (MSE) and their application in technical actuator systems. MSE consist of an elastic matrix containing suspended magnetically soft and/or hard particles. Additionally, they can also contain silicone oil, graphite particles, thermoplastic components, etc., in various concentrations in order to tune specific properties such as viscosity, conductivity and thermoelasticity, respectively. The focuses of investigations are the beneficial properties of MSE in prototypes for locomotion and manipulation purposes that possess an integrated sensor function. The research follows the principle of a model-based design, i.e. the working steps are ideation, mathematical modelling, material characterization as well as building first functional models (prototypes). The developed apedal (without legs) and non-wheeled locomotion systems use the interplay between material deformations and the mechanical motion in connection with the issues of control and stability. Non-linear friction phenomena lead to a monotonous forward motion of the systems. The aim of this study is the design of such mechanical structures, which reduce the control costs. The investigations deal with the movement and control of 'intelligent' mechanisms, for which the magnetically field-controlled particle-matrix interactions provide an appropriate approach. The presented grippers enclose partially gripped objects, which is an advantage for handling sensitive objects. Form-fit grippers with adaptable contour at the contact area enable a uniform pressure distribution on the surface of gripped objects. Furthermore, with the possibility of active shape adaptation, objects with significantly differing geometries can be gripped. To realise the desired active shape adaptation, the effect of field-induced plasticity of MSE is used. The first developed prototypes mainly confirm the functional principles as such without direct application. For this, besides the ability of locomotion and manipulation itself, further technological possibilities have to be added to the systems.
\end{abstract}

\footnotetext{
*Corresponding author: Jhohan Chavez, Department of Mechanical Engineering, Technical Mechanics Group, TU Ilmenau, Ilmenau, Germany, e-mail: jhohan-harvey.chavez-vega@tu-ilmenau.de

Valter Böhm, Ostbayerische Technische Hochschule Regensburg, Fakultät Maschinenbau, Regensburg, Germany

Tatiana I. Becker, Simon Gast, Igor Zeidis and Klaus Zimmermann, Technische Universität Ilmenau, Technische Mechanik, IImenau, Germany
} 
The first steps are therefore being taken towards integrated MSE based adaptive sensor systems.

Keywords: apedal locomotion systems, field-controlled particle-matrix interaction, field-induced plasticity, form-fit gripper, integrated sensor-actuator systems, magnetosensitive elastomer

\section{Introduction}

Both in macrotechnology (mechanical engineering, automotive engineering, precision engineering) and in particular in microtechnology (minimally invasive medical technology, microbiology), technical solutions are sought which are essentially determined by multifunctional materials and structural elements with dynamically variable functions. The technical basis is no longer formed by structurally delimitable modules with separate basic functions for the processing of energy, material and information. Rather, there is a far-reaching functional integration that begins with the material and is defined by it. Due to the great progress made in the fields of numerical mathematics, control and information technology (hardware and software), nonlinear effects in material behaviour that have been "rather combated" nowadays hardly pose any problems in application. On the contrary, nonlinear behaviour, anisotropy with respect to elongation, viscosity, magnetisation and, generally speaking, controllable material properties are desired in order to make completely new technical solutions possible. Magneto-sensitive elastomers (MSEs) belong to that class of smart materials whose mechanical properties can be controlled by means of magnetic fields. The MSE consist of an elastic matrix containing suspended magnetically soft and/or hard particles [1, 2]. Additionally, they can also contain silicone oil, thermoplastic components, graphite particles, etc. in various concentrations in order to tune specific properties such as viscosity [3-9], thermoelasticity [10, 11] and conductivity ([12] and the work of Monkman et al., Dielectric behavior of magnetoactive hybrid materials published in this special issue) respectively. Many investigations on MSE have been based on experiments using hard magnetic particles [13, 14]. For the investigations in this paper, the use of soft magnetic particles with a small degree of remanent magnetisation is preferable. The application of an external magnetic field results in a number of physical changes to the polymer including field stiffening and magnetostriction [15].

In the present work, the magnetically generated and controlled deformations are the central aspect in the application of MSE for locomotion (Section 3) and manipulation (Section 4). At the end of the 20th century, when nobody used the modern term 'soft robotics' yet, basic research began on the use of magnetically controllable materials (ferrofluid, magneto-rheological fluid, MSE, etc.) for the two tasks mentioned above in robotics. The technical implementation of locomotion based on the earthworm peristaltic using ferrofluid was first published by Saga \& Nakamura $[16,17]$. At 
about the same time as the authors, Kimura [18] investigated the use of ferroelastomers for locomotion purposes. Also the work of Zrinyi [19, 20] and Kashima [21] provided the experimental basis for mobile robots from exclusively compliant structures.

In the just mentioned papers, the experimental part dominates the research. Naletova and Zeidis [22-25] developed mathematical-mechanical models for wormlike locomotion systems made of MSE. They used methods of continuum mechanics and mechanics of rigid-body systems (especially asymptotic methods of non-linear mechanics).

Sensors (e.g. accelerometer) based on materials with magnetically controllable parameters were introduced in 2008 by Phan [26]. Petterson in 2010 described and evaluated a robot gripper that utilises the effects of a magnetorheological fluid [27]. The property of the so-called field-induced plasticity [3, 28] makes possible to use MSE in gripper systems. Chavez [5, 29] developed a form-fit gripper, which is described in detail in Section 4.

\section{Experimental results on material characterization}

When a magnetic field is present in the MSE, the iron particles tend to align with the lines of the magnetic field flux $[15,30]$. By removing the applied magnetic field, the particles return to their original position within the elastomeric matrix. The magnetic remanence, as well as the plastic deformation, after removing small or moderate magnetic field is negligible. In the experiments performed, the MSE samples contain silicone oil to provide a higher viscosity and allow the magnetic particles embedded in the MSE to move more freely, so greater effects of the influence of the magnetic field on the mechanical behaviour can be observed. In addition, the grade of compliance of the MSE with silicone oil in its original state before the application of the magnetic field is greater. This is an attractive property for applications in soft robotics (specifically in form-fit grippers). In order to study the mechanical behaviour of MSE materials, several test pieces are created for compression and tensile experiments. The experimental results enable to calculate an E-modulus. The E-modulus varies depending on the volume concentration of magnetic particles. In the manufacturing of MSE samples, Neukasil RTV 26 elastomer was used as a basis component mixed with the cross-linking agent A7. For a purely elastomeric specimen of this material, the hardness obtained is 7 in the scale Shore A. However, as mentioned above, the elastomeric matrix may contain a proportion of silicone oil to provide custom properties to the material. From previously presented studies [31-33], it is concluded that the recommended volume concentration of silicone oil in the specimen is $45 \%$. In the present work, the volume fraction of the silicone oil is calculated for the polymeric part of the MSE and not for the global volume. The polymeric part comprises the base of pure elastomer and silicone oil. Therefore, as an example, for the supposed case in which an MSE specimen has $50 \mathrm{vol} . \%$ of iron, the other $50 \mathrm{vol} . \%$ of the volume corresponds to the polymeric part. 


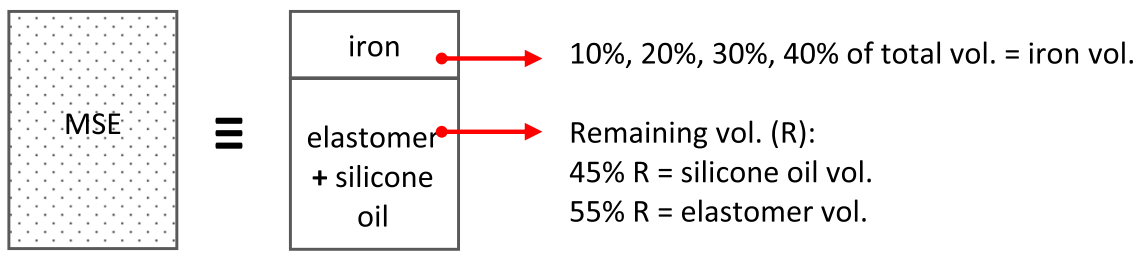

Figure 1: Volume distribution of the components of the MSE.

Since the silicone oil is $45 \%$ of the elastomeric part, the fraction of silicone oil is 22.5 vol.\% of the total volume of the MSE. This is graphically explained in Figure 1.

The ferromagnetic material used is carbonyl iron powder (CIP) grade CEP CC. The purity according to manufacturer data (BASF) is $99.5 \%$, and the average particle size lies between 6 and $10 \mu \mathrm{m}$. Cylindrical samples are manufactured for the compression (diameter: $16 \mathrm{~mm}$, height: $12 \mathrm{~mm}$ ) and tensile (diameter: $5 \mathrm{~mm}$, height: $80 \mathrm{~mm}$ ) tests. These geometries are shown in Figure 2. Additionally, samples for tensile tests have "heads" which are useful for clamping during the test, but do not have a large influence on the force measurement during the elongation. The iron concentration of the MSE samples varies from 10 to 40 vol.\%.

Four samples are manufactured and tested for the same volume concentration of iron, and the results shown in this section correspond to the average value obtained for these four samples. Each specimen is tested four times. The highest coefficient of variation found for the experimental results is $2.5 \%$, which confirms the repeatability of the experiments and the homogeneity of the samples.

For the compression tests, the samples are deformed up to 0.25 strain with $0.2 \mathrm{~mm} /$ $\mathrm{s}$ as recommended by ISO 7743. The deformation is performed under different magnetic field conditions. The magnetic field present in the specimens is not uniform. It has a gradient since a permanent magnet is used as a source (Figure 3). The permanent magnet is a Neodymium cylinder (diameter: $70 \mathrm{~mm}$, height: $35 \mathrm{~mm}$ ), magnetisation N45, Nickel-plated coated, residual magnetism (Br): 1.32-1.37 T, coercive field strength (bHc): 860-995 kA/m. The permanent magnet creates a magnetic field gradient where the intensity decreases at a greater distance from the magnet. For simplicity, a constant magnetic field for the whole cylinder volume of MSE is considered. This constant value is defined by the averaged magnetic field in vertical component. This simplification implies an idea of uniform magnetic field for the MSE materials. For tensile tests, the samples are only tested without the presence of magnetic fields, since their dimensions are more significant. On these samples, the gradient of magnetic field is greater when using the magnet as a source.

The results for tensile and compression test on the MSE samples are shown in Figure 4. From recommendations of standard norms, tensile tests comprehend a greater strain than compression tests. However for elastomers with particles located within the elastomer matrix, a large elongation concludes in breaking off the samples. 


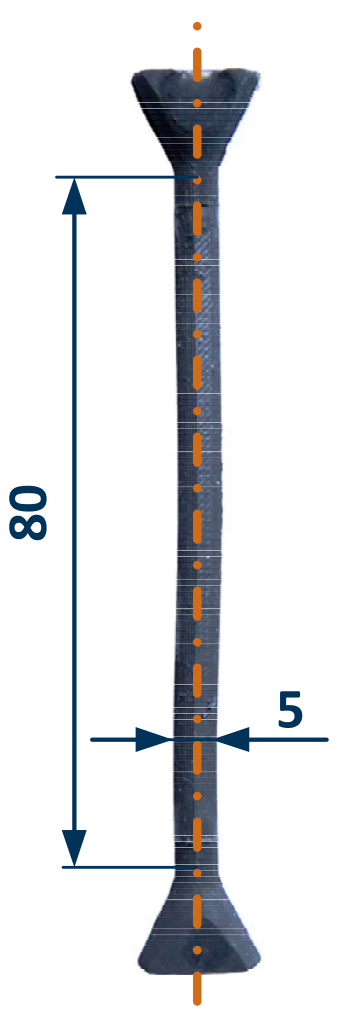

(a)

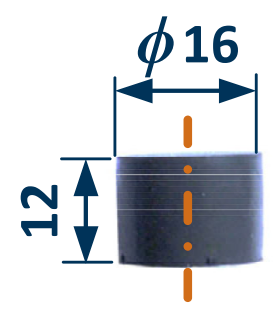

(b)
Figure 2: MSE cylindrical samples for tensile (a) and compression (b) tests.

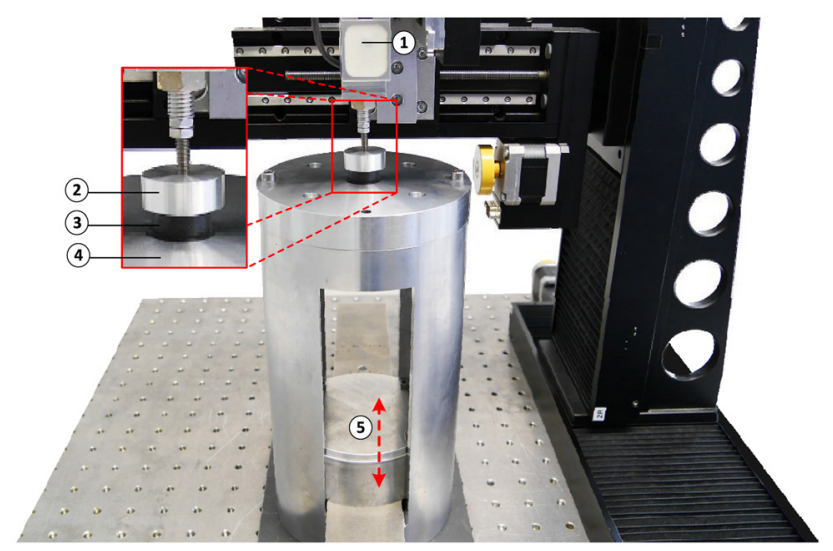

Figure 3: Experimental setup for compression of MSE cylindrical samples in presence of a magnetic field produced by permanent magnet. Its magnitude varies with distance. 1: load cell, 2: upper plate for compression, 3: MSE sample, 4: bottom plate for compression, 5: permanent magnet. 


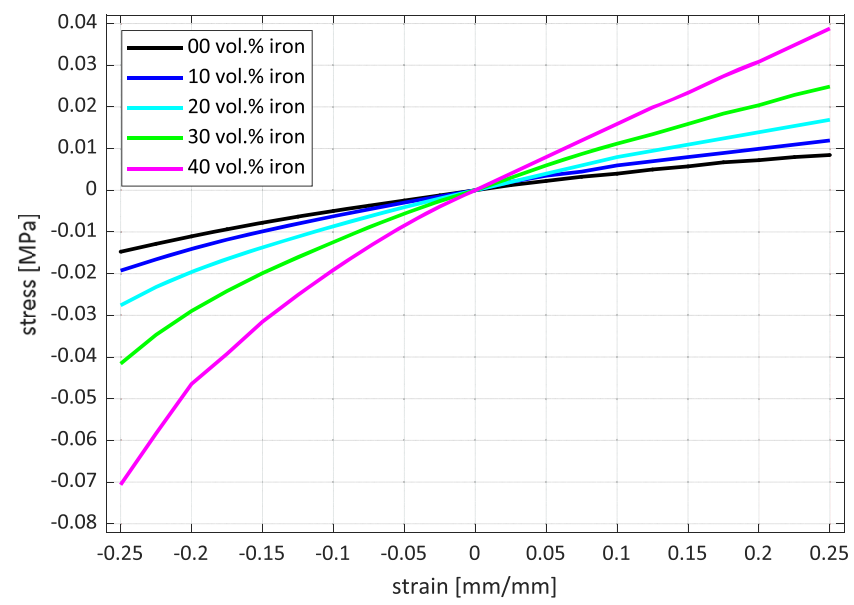

Figure 4: Results of tensile and compression test on the MSE samples, in absence of a magnetic field.

Thus, in this work results are presented for the same level of strain for both tensile and compression, to avoid the rupture of the samples. The positive and negative values of strain and stress correspond to tensile and compression, respectively. Due to the viscoelastic properties of the MSE materials, every compression or tensile experiment exhibits a hysteresis. However, for this work only the results for the increase of deformation are shown. Results for the relief of deformation are omitted since the experiments are performed with a quasi-static deformation.

As expected, samples with a bigger volume fraction of iron lead to a bigger stiffness. Additionally, pure polymeric samples (elastomer base and silicone oil) are also tested and their results are shown as a black curve in Figure 4. These samples do not experience a field-induced stiffness in presence of a magnetic field, since they do not contain ferromagnetic particles.

If the material is modelled with a linear behaviour, the E-modulus can be calculated as the ratio between the stress and strain. Nevertheless, as illustrated on the experimental results, a linear model suits only to the tensile part.

The results for 0.25 strain compression tests with a magnetic field present are summarized in Figure 5. The compression is realised in the presence of four different magnitudes of the magnetic field, which depends on the distance between the permanent magnet and the MSE sample (Figure 3). It is notable that the required force for the same deformation becomes greater as the magnetic field increases. This is very favourably used for form-fit grippers based on MSE and explained in Section 4. For a higher concentration of iron particles, the effect of field-stiffening is amplified. Samples with 30 and 40 vol.\% iron are the most beneficial for further investigations. Nonetheless, the ease of manufacturing MSE materials decrease for smaller amount of pure elastomer part. Therefore, MSE materials with $30 \mathrm{vol} . \%$ are considered for the next paragraphs. 


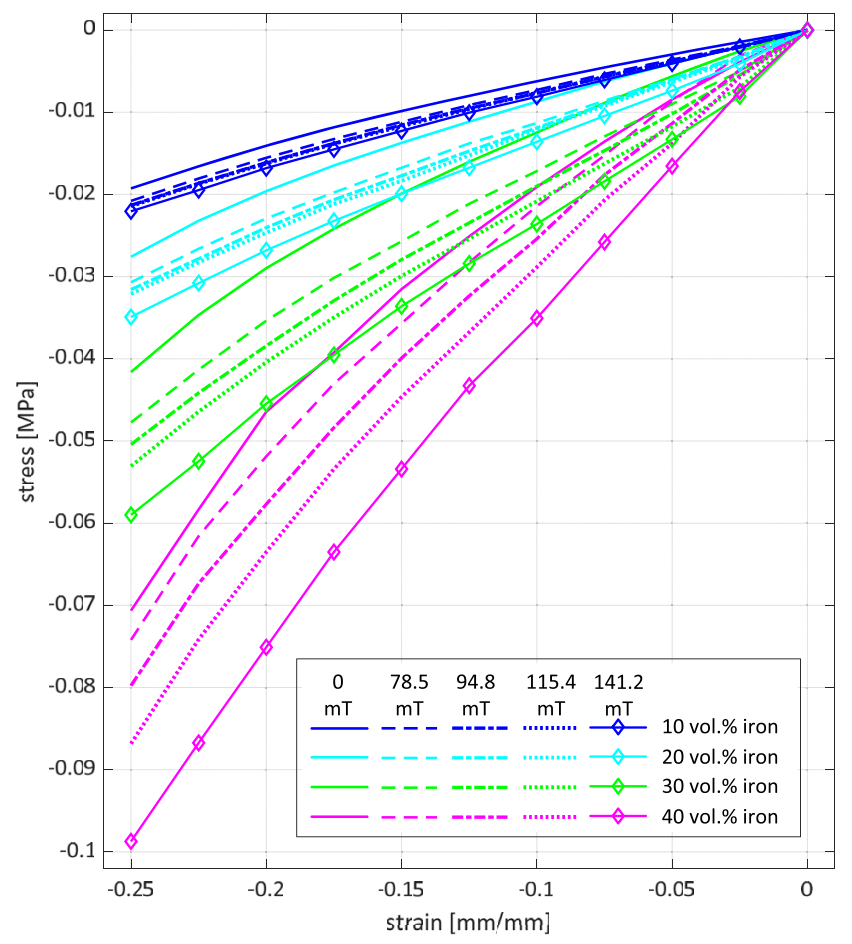

Figure 5: Results of compression test on the MSE samples, with four different magnitudes of the magnetic field.

A linear material behaviour is a poor model for the MSE. A hyperelastic model is more appropriate for the MSE. For simplicity the chosen model is Neo-Hookean since it comprehends a single parameter (initial shear modulus). To obtain the parameter for the material model, the compression experiments are simulated using finite element (FE) tools (Ansys Workbench ${ }^{\circledR}$ ). After a few iterations, a parameter for the material model which enables a proper fit with the experimental results is found. Figure 6 shows the experimental and simulated results of compression for the MSE with $30 \mathrm{vol} . \%$ iron for three magnitudes of magnetic field. However, this material model also has disadvantages. The Neo-Hookean model does not conclude as a stress-strain curve with inflections as in experimental measurements and these inflections are becoming more pronounced for bigger magnetic fields. Nevertheless, the difference between stresstrain curves from simulations and experimental results is small compared to the measured forces. The most perceptible error is found in the results for compression tests developed in the presence of the greatest magnetic field (average of $141.2 \mathrm{mT}$ ) in MSE.

The parameters obtained for the material model are used in Section 4 for MSE based form-fit grippers. 


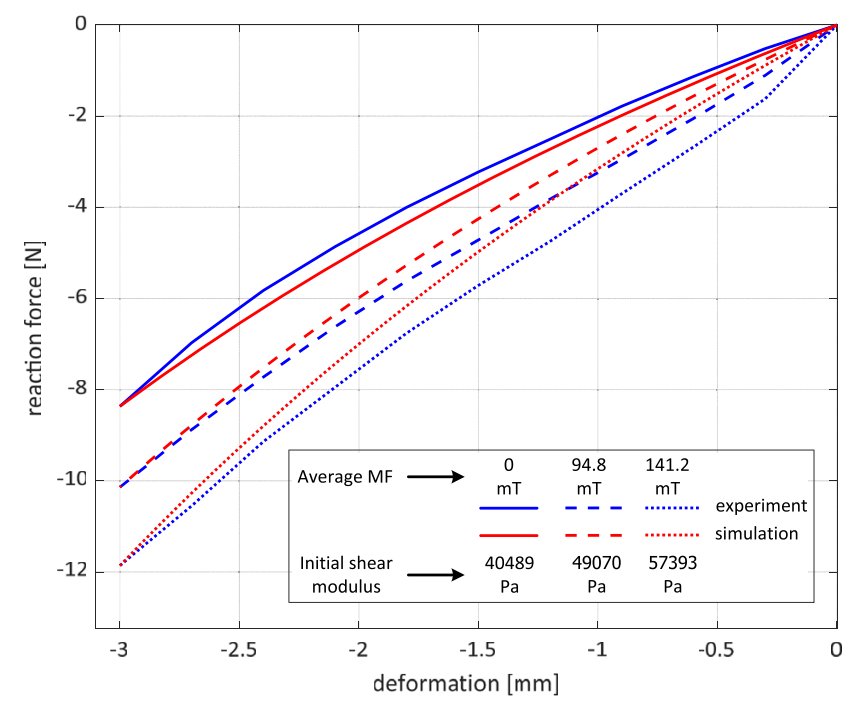

Figure 6: Results for compression on MSE with 30 vol.\% iron for three values of magnetic field. Blue curves: experimental results. Red curves: simulations using Neo-Hookean material model.

Borin et al. present on their work Magneto-mechanical properties of elastic hybrid composites in the present special issue, another approach to the properties of magnetic composites with different polymeric matrixes.

\section{Non-pedal locomotion systems based on MSE}

Basic research on the deformation of membrane-enveloped wormlike ferrofluid structures and on the behaviour of MSE materials in an external magnetic field forms the basis for the development of novel apedal locomotion systems. These locomotion systems, often inspired by biological examples [16, 24, 34], can be divided into two classes. Locomotion on free ferrofluid surfaces by a locally and temporally changing magnetic field is called passive, when a non-magnetic object is moved due to the deformation of this surface. With active locomotion systems, the movement is generated in the system itself. MSE objects experience a change of position, see Figure 7.

Such active systems form the primary goal of the research, with the design focus on the realisation of an apedal locomotion. A model-based approach leads to the development of systems whose locomotion is based on high-frequency excitation of MSE. By the specific control of different oscillation modes of the locomotion systems, the definition of the motion direction succeeds beside the adjustment of the velocity. Uniaxial and planar locomotion systems with only one electromagnetic drive are developed. 


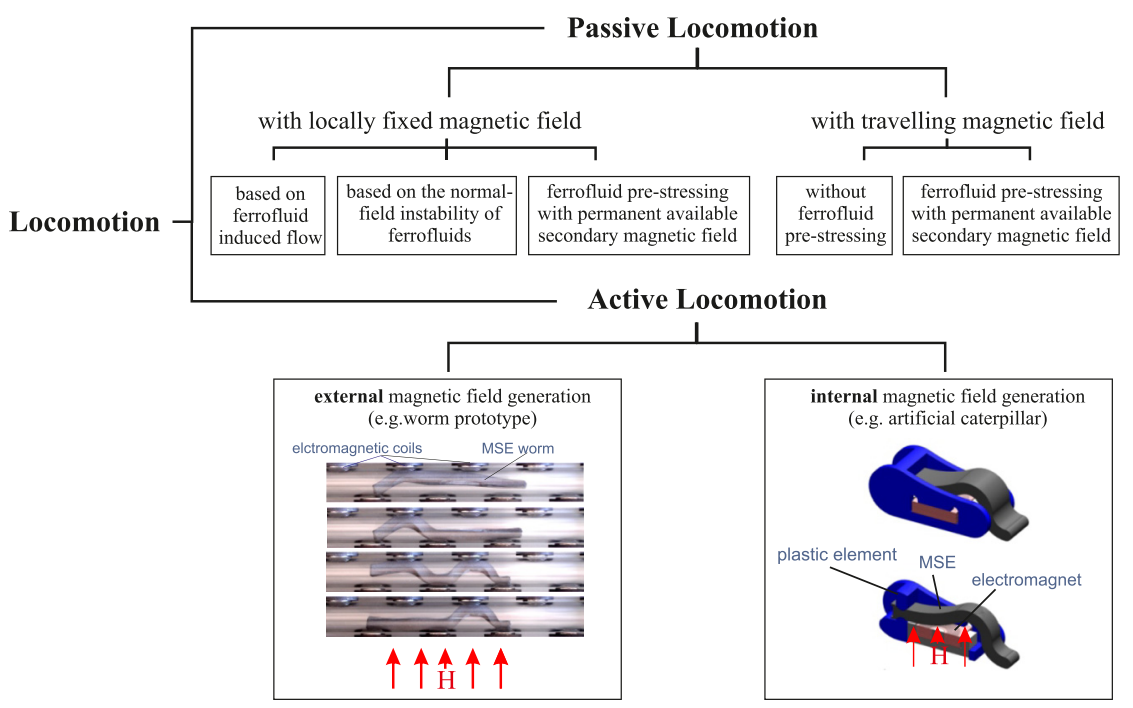

Figure 7: Active and passive locomotion systems realised with magneto-sensitive elastomer.

\subsection{A basic experiment with locomotive MSE}

In order to understand how magnetic field induced motion can be realised with MSE and whether this motion is stable in the mechanical sense, a basic experiment is considered. The system consists of two material points with masses $m$ connected by an MSE spring of stiffness $k$. Under the influence of an external magnetic field, which changes periodically, a harmonic internal force $F(t)$ is generated, see Figure 8 .

The motion of the system is governed by the differential equations.

$$
\begin{aligned}
& m \ddot{x}_{1}+k\left(x_{1}-x_{2}\right)=F\left(\dot{x}_{1}\right)+F(t), \\
& m \ddot{x}_{2}+k\left(x_{2}-x_{1}\right)=F\left(\dot{x}_{2}\right)-F(t),
\end{aligned}
$$

where $F(t)=F_{a}(1+\cos \psi)$, with $\psi=v t$. The Coulomb friction force acting on the material points with number $i$ yields

$$
F\left(\dot{x}_{i}\right)= \begin{cases}m g \mu_{-}, & \text {if } \dot{x}_{i}<0, \\ F_{0}, & \text { if } \dot{x}_{i}=0, \\ -m g \mu_{+}, & \text {if } \dot{x}_{i}>0\end{cases}
$$

where $\mu_{-}$and $\mu_{+}$are anisotropic friction coefficients with $\mu_{-} \geq \mu_{+} \geq 0$. The force $F_{0}$ satisfies the relation $-m g \mu_{+}<F_{0}<m g \mu_{-}$.

To reduce the number of parameters that characterise the system, the dimensionless variables are introduced 


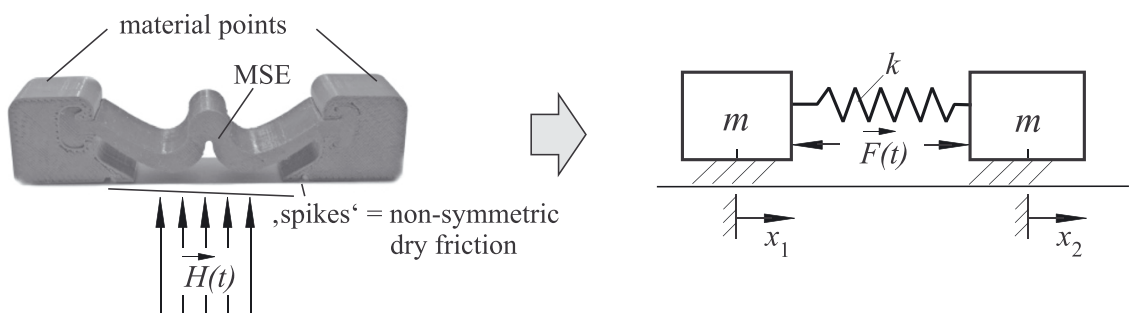

Figure 8: From the real system to the mechanical model.

$$
\begin{gathered}
x_{i}^{\star}=\frac{x_{i}}{L}, \quad t^{\star}=t \omega, \quad v^{\star}=\frac{v}{\omega}, \quad \dot{x}_{i}^{\star}=\frac{\dot{x}_{i}}{L \omega}, \quad F^{\star}=\frac{F}{m g \mu_{-}}, \\
\mu_{0}^{\star}=\frac{F_{0}}{m g \mu_{-}}, \quad \varepsilon=\frac{m g \mu_{-}}{k L}, \quad \alpha=\frac{F_{a}}{m g \mu_{-}}, \quad \mu=\frac{\mu_{+}}{\mu_{-}}(0 \leq \mu \leq 1),
\end{gathered}
$$

where $\omega^{2}=k / m$ and $L$ is the scale of length used for the procedure of nondimensionalisation.

Proceeding with the dimensionless variables in Equations (1) and (2) and then omitting the asterisks, identifying the variables $x_{i}^{\star}$ and $t^{\star}$, it follows

$$
\begin{aligned}
& \ddot{x}_{1}+x_{1}-x_{2}=\varepsilon\left[\alpha(1+\cos \psi)+r_{1}\right], \\
& \ddot{x}_{2}+x_{2}-x_{1}=\varepsilon\left[-\alpha(1+\cos \psi)+r_{2}\right],
\end{aligned}
$$

where

$$
r_{i}=\left\{\begin{array}{cc}
1, & \text { if } \dot{x}_{i}<0 \\
\mu_{0}, & \text { if } \dot{x}_{i}=0 \\
-\mu, & \text { if } \dot{x}_{i}>0
\end{array}\right.
$$

Assuming $\varepsilon \ll 1, \alpha \sim 1,\left|x_{2}-x_{1}\right| \sim 1$, the procedure of averaging [35] is applied to system (4). For this purpose new variables are introduced: the velocity of the centre of mass $V=\left(\dot{x}_{1}+\dot{x}_{2}\right) / 2$ and the deviation relatively to the centre of mass $z=\left(x_{2}-x_{1}\right) / 2$. Replacing $z=a \cos \varphi, \dot{z}=-a \sqrt{2} \sin \varphi, \varphi=t \sqrt{2}+\vartheta$, where $V, a, \vartheta$ - slow variables, system (4) takes the so-called standard form:

$$
\begin{aligned}
& \dot{V}=\frac{\varepsilon}{2}(F(V+a \sqrt{2} \sin \varphi)+F(V-a \sqrt{2} \sin \varphi)), \\
& \dot{a}=\frac{\varepsilon}{2 \sqrt{2}} \sin \varphi(F(V+a \sqrt{2} \sin \varphi)-F(V-a \sqrt{2} \sin \varphi)+2 \alpha(1+\cos \psi)), \\
& \dot{\varphi}=\sqrt{2}+\frac{\varepsilon}{2 a \sqrt{2}} \cos \varphi(F(V+a \sqrt{2} \sin \varphi)-F(V-a \sqrt{2} \sin \varphi)+2 \alpha(1+\cos \psi)), \\
& \dot{\psi}=v .
\end{aligned}
$$

The system (6) is investigated in a vicinity of the main resonance $v=\sqrt{2}+\varepsilon \Delta(\Delta \neq 0)$. Introducing a new slow variable $\xi=\psi-\varphi$, after averaging on a fast variable $\varphi$ it yields 


$$
\begin{aligned}
& \dot{V}= \begin{cases}\varepsilon, & \text { if } V<-a \sqrt{2}, \\
-\varepsilon\left(\frac{1+\mu}{\pi} \arcsin \frac{V}{a \sqrt{2}}-\frac{1-\mu}{2}\right), & \text { if }|V| \leq a \sqrt{2}, \\
-\varepsilon \mu, & \text { if } V>a \sqrt{2},\end{cases} \\
& \dot{a}= \begin{cases}-\frac{\varepsilon}{\sqrt{2}}\left(\frac{1+\mu}{\pi} \sqrt{1-\frac{V^{2}}{2 a^{2}}}+\frac{\alpha}{2} \sin \xi\right), & \text { if }|V| \leq a \sqrt{2}, \\
-\varepsilon \frac{\alpha}{2 \sqrt{2}} \sin \xi, & \text { if }|V|>a \sqrt{2},\end{cases} \\
& \dot{\xi}=\varepsilon\left(-\frac{\alpha}{2 a \sqrt{2}} \cos \xi+\Delta\right) .
\end{aligned}
$$

By the definition of the steady-state motion, the variables $V, a$ and $\xi$ are constant according to the averaged equations. From Equations (7-9) it can be found

$$
\begin{aligned}
V & =\frac{\sin \Phi}{|\Delta|} \sqrt{\frac{\alpha^{2}}{4}-E^{2} \cos ^{2} \Phi}, \quad \Delta \neq 0, \\
a & =\frac{1}{|\Delta| \sqrt{2}} \sqrt{\frac{\alpha^{2}}{4}-E^{2} \cos ^{2} \Phi}, \quad \alpha \geq 2 E \cos \Phi, \\
\sin \xi & =-\frac{2 E \cos \Phi}{\alpha}, \cos \xi=\frac{2 \Delta}{\alpha|\Delta|} \sqrt{\frac{\alpha^{2}}{4}-E^{2} \cos ^{2} \Phi,} \\
E & =\frac{1+\mu}{\pi}, \quad \Phi=\frac{\pi(1-\mu)}{2(1+\mu)} .
\end{aligned}
$$

The motion of the system with the velocity $V$ from Equation (10) is stable.

The result of the numerical integration of the exact system (4) is given in Figure 9. The following values of parameters for an MSE are taken: $m=3 \cdot 10^{-3} \mathrm{~kg}, k=3 \mathrm{~N} / \mathrm{m}$, $F_{a}=3 \cdot 10^{-3} \mathrm{~N}, v=47 \mathrm{~s}^{-1}, L=1 \mathrm{~cm}, \mu_{-}=0.1, \mu_{+}=0.05$. So, the values of the dimensionless parameters are: $\varepsilon=0.1, \mu=0.5, \alpha=1, \Delta=0.8$.

The first equation of Equation (10) gives the value for the dimensionless velocity of the centre of mass $V=0.17$. This value corresponds to the dimensional velocity $V=5.4 \mathrm{~cm} / \mathrm{s}$.

For a nonlinear spring with a small cubic nonlinearity, the expression for the spring force $F_{e l}$ in dimensionless variables is

$$
F_{\mathrm{el}}=-\chi-\varepsilon \beta x^{3}, \quad \beta \sim 1,
$$

here $\beta$ is the nonlinearity measure. In this case the expression for the stationary velocity $V$ is 


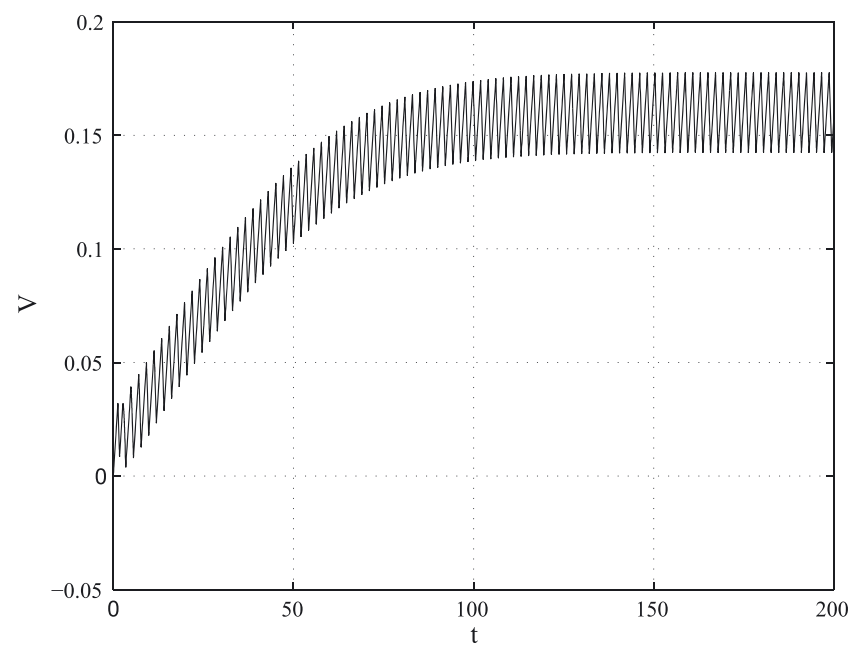

Figure 9: The velocity $V$ of the centre of mass vs. time $t$, following from the numerical integration of the exact equations of motion. The stationary velocity is nearly equal to 0.17 .

$$
V=a \sqrt{2} \sin \Phi
$$

and the amplitude $a$ is determined from the equation

$$
a\left|3 a^{2} \beta-\Delta \sqrt{2}\right|=\sqrt{\frac{\alpha^{2}}{4}-E^{2} \cos ^{2} \Phi} .
$$

Remark: In the investigations given above, the motion with a periodic excitation force, which has a zero mean value, is considered. In this case, the motion of the centre of mass requires asymmetry. Here, this asymmetry is achieved due to different friction coefficients, when changing the direction of motion (anisotropic surface). The internal force itself is assumed to be harmonic (i.e. symmetrical). The motion is only possible in the direction of less friction. In [36] a two-material point system with different masses is considered. There, the variable part of the periodic excitation force is considered asymmetrical, provided that it has zero average (e.g. increase and decrease of the magnetic field occur at different velocities). It is shown that in this case it is possible moving not only on an isotropic surface, but even on an anisotropic one to the side of more friction.

Thus, by changing the magnetic field, it is possible to control not only the velocity of the system, but also the direction of motion, see Section 3.2. 

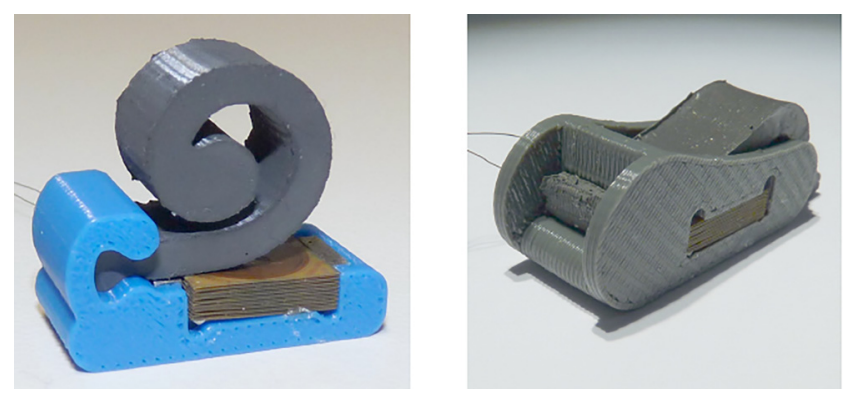

Figure 10: Vibration driven locomotion systems for rectilinear motion based on MSE - 'Type 1' (left) and 'Type 2' (right).

\subsection{Vibration driven mobile systems for rectilinear motion}

For rectilinear uni- and biaxial motion, two vibration-driven locomotion systems based on MSE are developed. To explore the mechanical properties of MSEs under magnetic fields, please refer to the work Magnetoactive elastomers for magnetically tunable vibrating systems written by Becker et al. and published in this special issue. The locomotion of these systems (Figure 10) is caused by a periodic deformation of a compliant MSE body. The deformation is generated by integrated micro-coils. A periodically alternating magnetic field causes an oscillation of the MSE. The friction force between the supporting surface (ground) and the system changes periodically due to a change of the normal force. The oscillation mode can be varied in dependence of the pulsing frequency in a wide range.

The qualitative description of the mechanical performance of the two systems is provided by transient dynamical analyses by means of the FE-Software ANSYS ${ }^{\circledR}$, see Figures 11 and 12 for the simulation of 'Type 1'.

Figure 13 shows the velocity at various driving frequencies ranging from 1 to $30 \mathrm{~Hz}$ when the robot is actuated using forces $F=0.006 \mathrm{~N}$ (top, blue) and $F=0.010 \mathrm{~N}$ (bottom, green). The FE-simulations confirm the result published in [36] that the direction of movement of the robot and its velocity depend essentially on the relation between the eigenfrequency of the system and the driving frequency. There are multiple driving frequency ranges, which cause the robot locomotion in opposite directions from the initial position. At $F=0.010 \mathrm{~N}$, the robot moves in the positive $x$-direction at the frequency ranges between $5 \ldots 6 \mathrm{~Hz}$ and $10 \ldots 13 \mathrm{~Hz}$, and in the negative $x$-direction between $6 \ldots 10 \mathrm{~Hz}$ and at over $13 \mathrm{~Hz}$, Figure 13. At low frequencies, the internal force is nearly equal to the excitation force. At higher driving frequencies, particularly in the vicinity of the natural frequencies, a marked change in the characteristics of the internal forces is observed. The system 'Type 1' achieves speeds in the range of $2 \ldots 3 \mathrm{~mm} / \mathrm{s}$.

In contrast to 'Type 1', the locomotion system 'Type 2' converts directly the forced vibrations of the MSE into a propulsive force due to its contact with the ground 

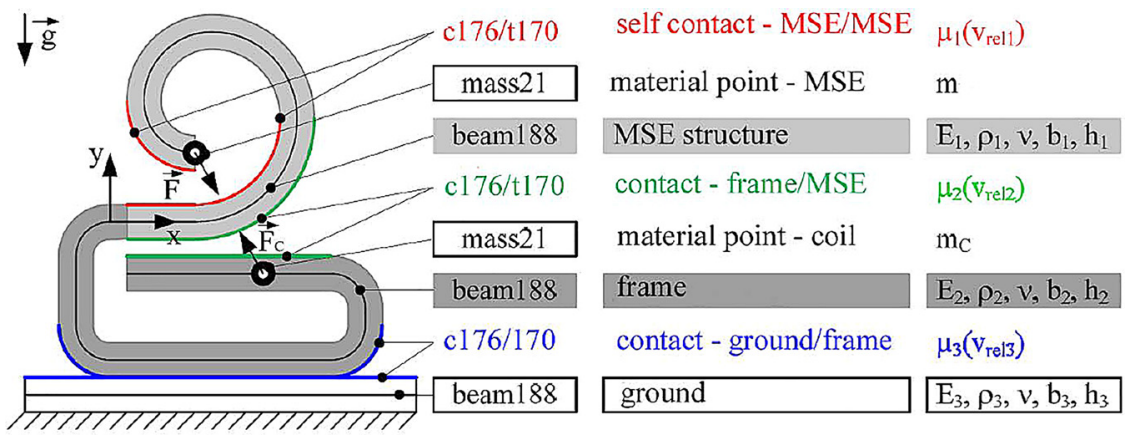

Figure 11: The mechanical model of the biaxial locomotion system 'Type 1' with ANSYS-elements used in simulation and the corresponding parameters.

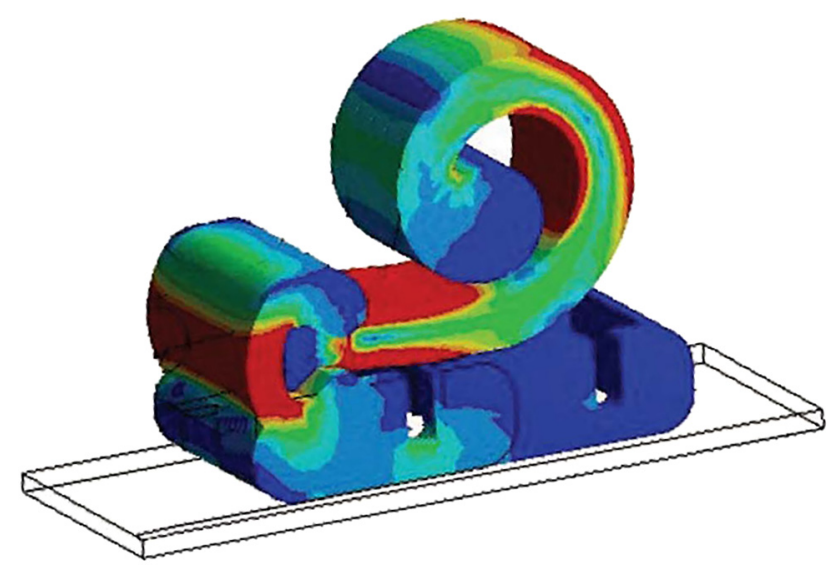

Figure 12: The equivalent stresses after von Mises for the locomotion system 'Type 1'.

(Figure 14, left). Only uniaxial locomotion is possible. The images from a high-speed camera show selected motion sequences with and without contact of the MSE to the ground (Figure 14, right). Depending on the friction pairing between the elastomer and the ground, the maximum velocity achieved with 'Type 2' is about $80 \mathrm{~mm} / \mathrm{s}$. Once again, there is great potential for optimisation both in the shape of the MSE and in the control via the periodically changing magnetic field of the miniature coil.

\subsection{Amoeboid locomotion systems based on MSE}

Conventional terrestrial locomotion systems with legs or wheels have a limited field of application, and they are difficult to miniaturize. Therefore, for planar motion biologically inspired locomotion systems following the paradigm of an amoeba are 

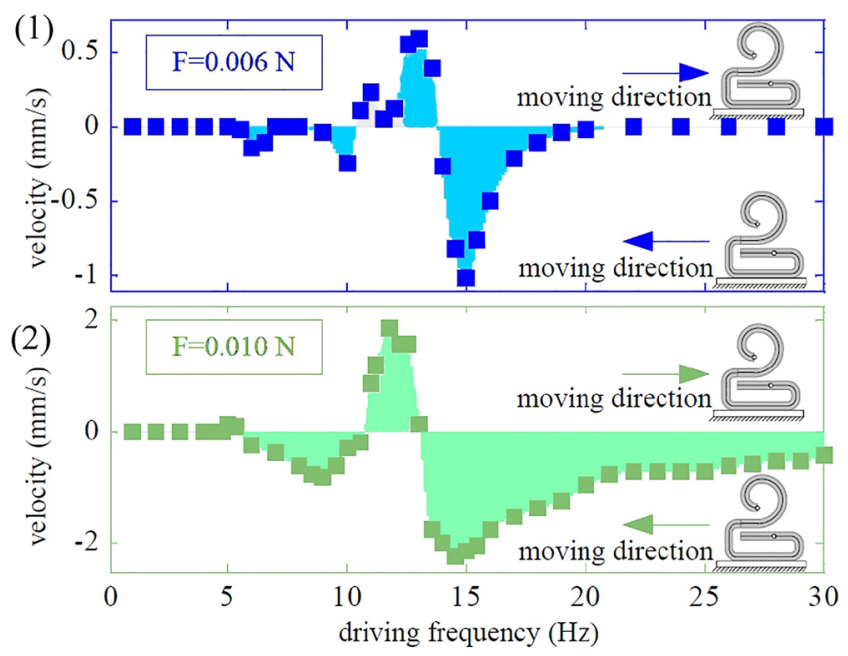

Figure 13: Velocity of the robot in dependent on the driving frequency for the actuating forces (1) $F=0.006 \mathrm{~N}$ (blue) and (2) $F=0.010 \mathrm{~N}$ (green).
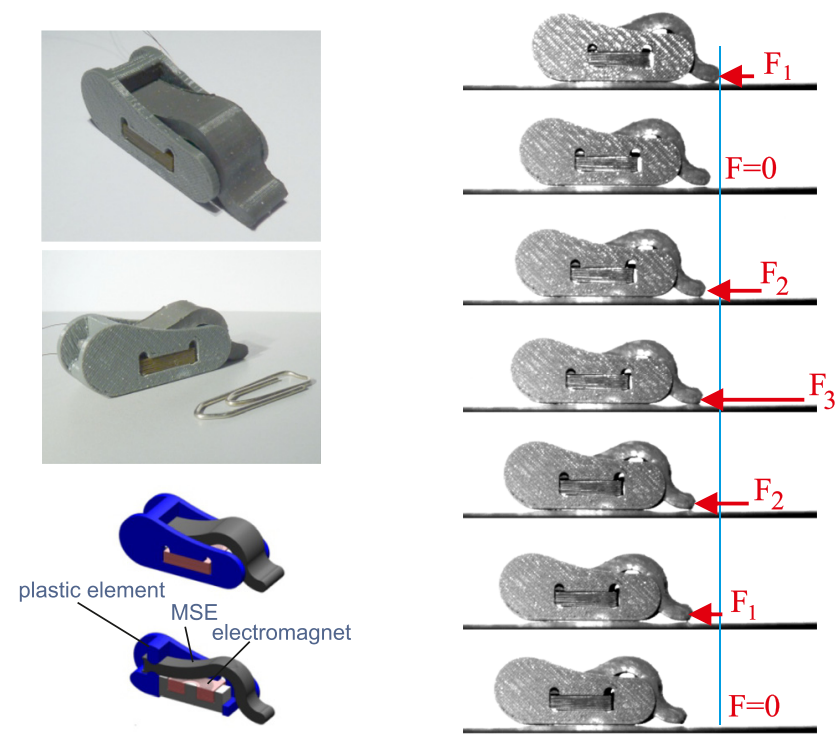

Figure 14: Apedal locomotion system 'Type 2' with parameters. Mass: $7.3 \mathrm{~g}$, length: $37 \mathrm{~mm}$, width: $12 \mathrm{~mm}$, height: $13 \mathrm{~mm}$ (left); and motion sequences with driving forces (right).

developed, which use MSE and controllable by magnetic fields. The movement of a natural amoeba is characterised by cytoplasmic streaming and continuous changing of the body shape [34, 37]. The periodic change of the shape of the technical locomotion system is also realised by means of a vibration drive using permanent magnets and 

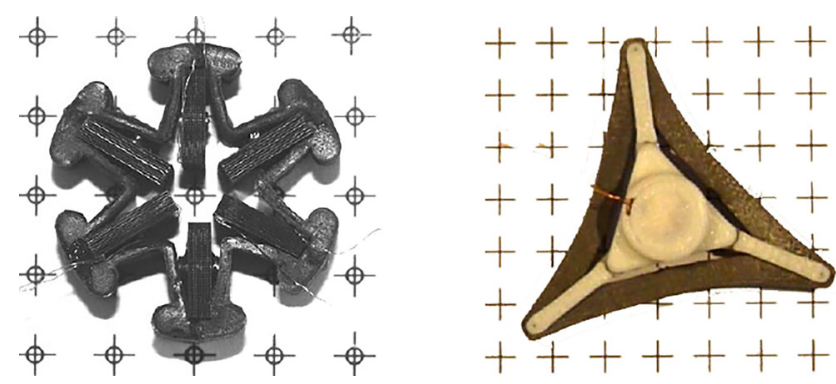

Figure 15: Planar locomotion systems based on MSE: the biological inspired prototype 'Moving star' (left) and the mobile system 'Triangular amoeba'(right).

miniature coils. According to the classification in Figure 7, these are active locomotion systems, Figure 15.

Using the example of the 'Moving star', the development process from the biological example (amoeba proteus) via the mechanical model to the technical amoeba is shown in Figure 16. From the point of view of mechanics, the amoeba means the realisation of a fluid flow (caused by pressure gradients) and the change in the flexibility of a body with variable contours. In the technical implementation, the forces are not generated by pressure differences but magnetically. The electromagnetic driving force is generated between activated and non-activated coils and the envelope body made of MSE. This driving force generates a locally differently pronounced deformation of the flexible body. In this area, the static friction force between the locomotion
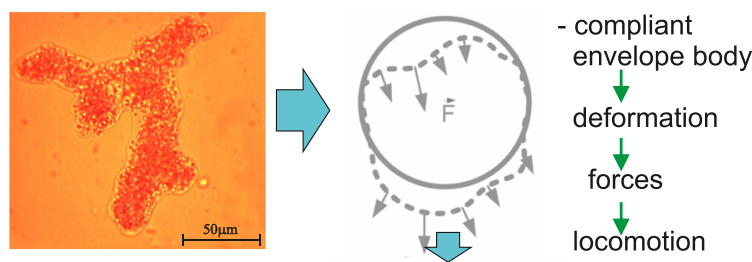

amoeba proteus

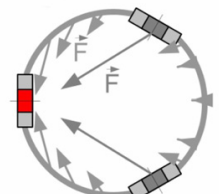

- magnetic compliant envelope body (MSE)

- coils with iron core
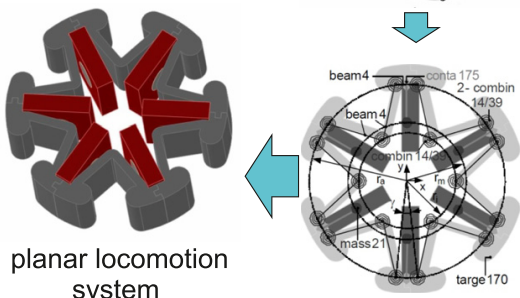

- increasing the number of coils

- coil arrangement closer to each other

planar locomotion system

- controllable magnetic field

Figure 16: From the biological example to the prototype (photo of amoeba proteus by Danja Voges $\dagger$ ). 
system and the ground is locally exceeded. Thus, a periodic driving force leads to the locomotion of the system.

With the coils of size $11.5 \times 11.5 \times 3 \mathrm{~mm}$ integrated in the MSE and arranged at $60^{\circ}$ to each other, a sufficiently close relative position to each other is achieved. This leads to an increase in the electromagnetic forces and ensures the energy efficiency during motion. The direction of motion of the system is determined by the choice of coils activation. The electromagnets each have an iron core. The flexibility of the MSE body is ensured on the one hand by the material itself, and on the other hand by the shape of the body. The connecting elements between the coil carriers are designed as joints with distributed compliance [38].

A model-based simulation of the system performance is provided by transient dynamical analyses by means of FE-Method. Within the model, the micro coils are treated as material points. Their connection to the compliant body is ensured by rigid beam elements. The MSE body, with a diameter of $40 \mathrm{~mm}$ is assembled with beam elements in the range of great curvature and with discrete spring-damper-elements at the intersection of different cross areas. The actuating force is represented by single forces acting on each mass point. The value and the direction of these forces depend on the relative position of the coils and, of course, the control algorithm. Forces acting on the compliant body are neglected due to the small permeability of it. Figure 17 (right) shows exemplarily the obtained results for the following case: two coils A and B in Figure 17 (left) are actuated simultaneously and alternate periodically. The system moves in $y$-direction. The focus is on the velocity of the centre point of the system in dependence on the actuating frequency. It can be seen, that the direction of motion can be controlled by means of the actuating frequency. The maximum average velocity of the 'Moving star' is $5 \mathrm{~mm} / \mathrm{s}$ at the driving frequency of $22 \mathrm{~Hz}$. The results of the numerical simulation using FE-Method and the measurements during the experiment are in good qualitative and quantitative agreement.

In the prototype 'Triangular amoeba', the idea of realisation of a simple actuation is based on the use of permanent magnets, which are attached to the shaft of a rotary drive inside the MSE structure. The dynamic excitation of the MSE takes place by means
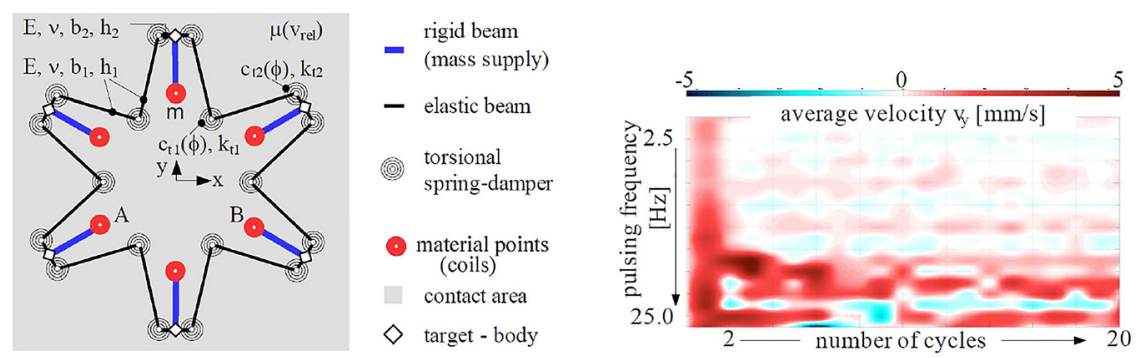

Figure 17: The model elements for the FE simulation with parameters (left) and the velocity in $y$-direction depending on the pulsing frequency (right). 


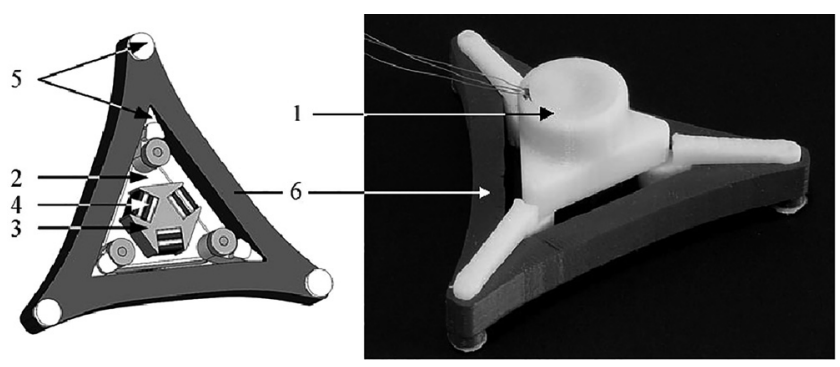

Figure 18: Structure of the locomotion system 'Triangular amoeba'. 1: motor, 2: housing, 3: rotor, 4: permanent magnet, 5: plastic body, 6: MSE.

of an alternating magnetic field. The MSE structure reacts only passively to a magnetic field and does not operate actively as an actuator. To be able to move in the plane, the system uses an asymmetric geometry of the compliant MSE. The structure consists of addition-curing silicone with a Shore hardness A of 8 . The mass ratio of elastomer to CIP (same particles as presented in Section 2) is 3:1 for the MSE part. The realised prototype has an outer diameter of $67.5 \mathrm{~mm}$ and a height of $23 \mathrm{~mm}$. The mass of the system is 21.6 g, Figure 18.

Modal analyses and transient motion analyses based on a multibody system model are performed to describe the locomotion system. Figure 19 (top) shows the excitation control forces at the points A, B and C of the MSE. The results of an exemplary selected transient simulation are given in Figure 19 (bottom, right). At an excitation frequency of $1.5 \mathrm{~Hz}$, the system performs a rotary motion. The theoretical results agree with the experimental data and show that with the mentioned type of control and the selected asymmetrical basic MSE structure, a locomotion in the plane is possible.

\section{Form-fit grippers}

The use of MSE in form-fit grippers is of great interest in the field of soft robotics [12,31, 32, 39]. Depending on the elastomeric base and the amount of silicone oil added, the material can be soft in its original state and allow a higher adaptability when deformed by objects with certain geometries. After applying a magnetic field, the MSE becomes harder due to the field-induced stiffening effect. The increase of its stiffness after the application of the field is favourable as it keeps the deformation (and therefore the geometry) from the object to grip.

A scheme for this manipulation system is shown in Figure 20. At the beginning (i) the MSE based grippers are found in their original state and are soft, so they can be deformed by flexible objects without the need to exert a great force (ii). After switching on a magnetic field in the vicinity of the MSE end-effectors, the rigidity of the MSE material increases and due to the field-induced plasticity the deformation can be 

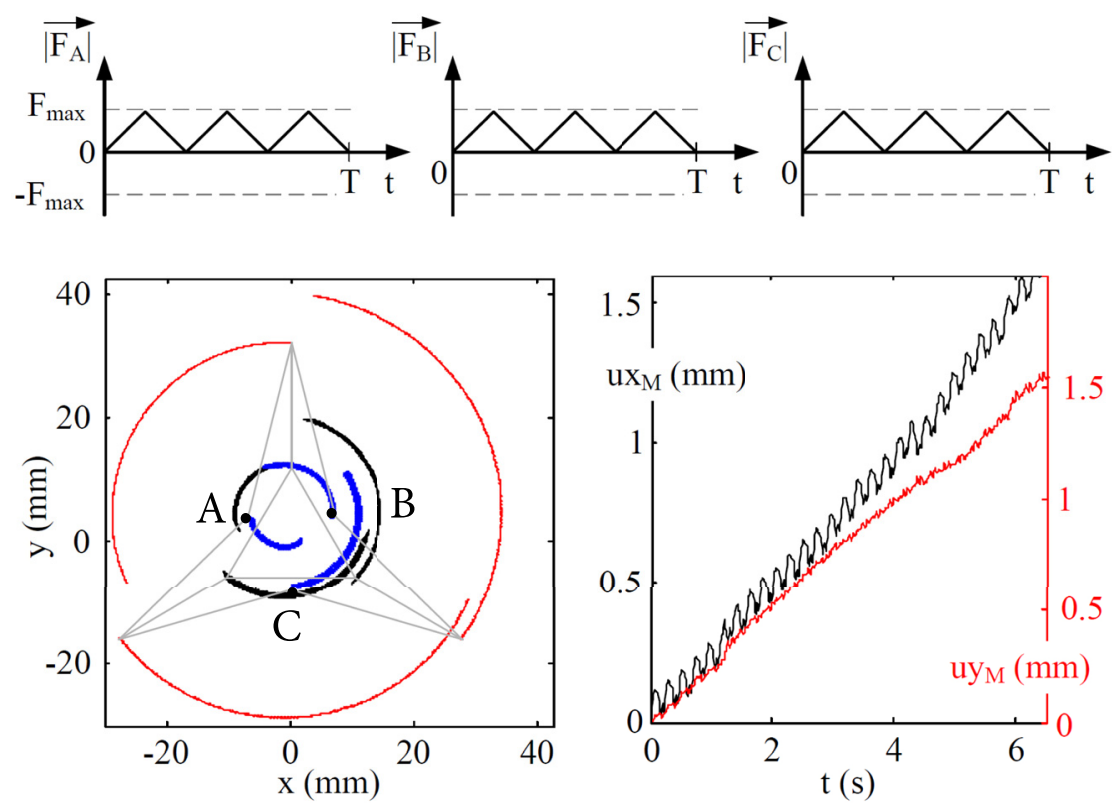

Figure 19: Applied control functions of the forces in the points A, B and C (top) and paths of the nodes after 100 actuation periods for an excitation frequency of $f=1.5 \mathrm{~Hz}$ with associated movement of the centre during a circular motion of the system (bottom).

recorded (iii). The shape adaptability depends on the composition of the MSE and its geometry. As long as the magnetic field is not removed, the deformation will remain intact in the MSE end-effectors, even when the object to grab is removed (iv). This can be used to transport elements with the same geometry ( $\mathrm{v} . . n-1)$. Finally, by removing the magnetic field, the mechanical properties are almost entirely recovered, without a noticeable plastic deformation ( $n$ ). To develop an MSE end-effector capable to grip various shapes, the investigations are focused on three complex end-effector shapes. These prototypes are fabricated and tested with two objects in order to examine the quality of their shape adaptability. The deformations are simulated by using the mechanical behaviour models introduced in Section 2. Previous studies show that promising geometries for the final effectors are hollow rotational-symmetric bodies. The hollow half spherical MSE end-effector has a thinner MSE layer in the area in contact with the object to grip in order to allow compliance on the deformation [32], see Figure 21a. The half spherical MSE is modified and two new geometries are presented, both with a cylinder-like shape but each with different curvatures in their designs. The most relevant geometric details on the design of the three mentioned MSE prototypes are shown in Figure 21.

The second studied geometry (Figure 21b) is named as a cylindrical-convex geometry, since the lateral layer (green dotted curve) corresponds to an elliptical 


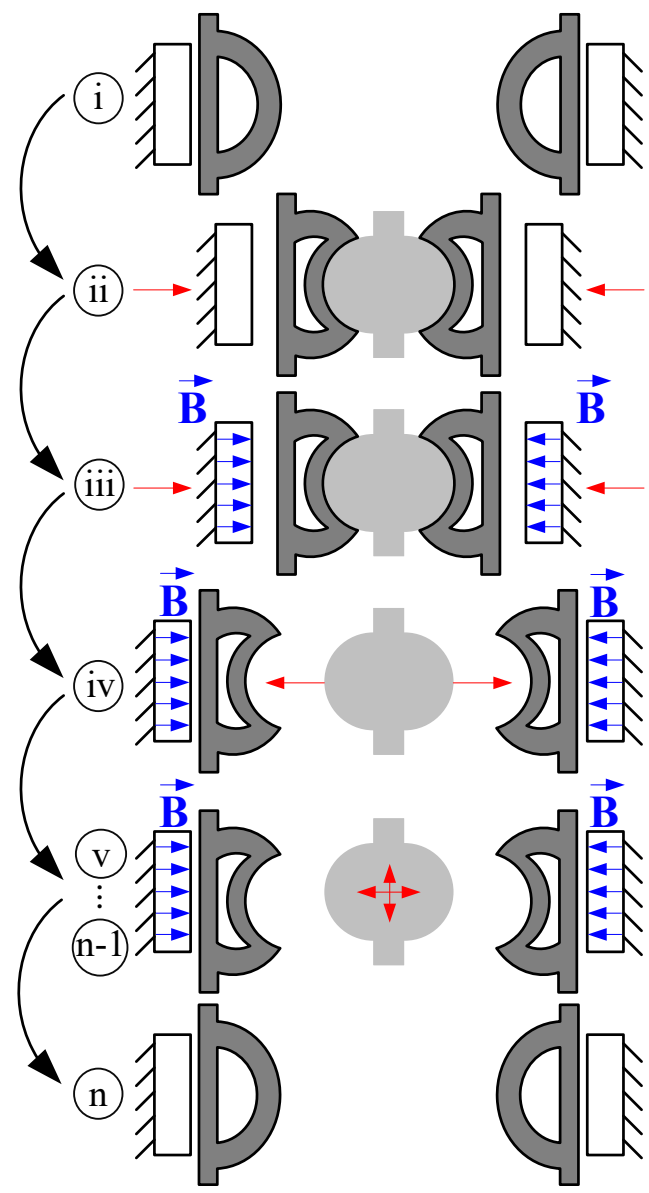

Figure 20: Gripping process by using a form-fit MSE based end-effector.

geometry, which centre lies outside of the MSE sample. The third geometry (Figure 21c) is a cylindrical-concave sample and differs from the second on the lateral face. In this case the centre of the elliptical geometry lies inside the MSE sample. For the fabrication of the samples, the composition studied in detail in Section 2 (30 vol.\% iron and $45 \mathrm{vol} . \%$ silicone oil for the polymeric part) is preferable.

The steps (i-iii) from the gripping process presented in Figure 20 are of interest in the present work in order to compare the shape adaptability of the three MSE endeffectors. It is not possible to detect how good the shape adaptability of the MSE samples is, when the objects are still surrounded (see [ii], Figure 20). Nevertheless, the hyperelastic material model presented on Section 2 can be used to calculate an approximation of the deformations during the manipulation and compare qualitatively this simulation with the experimental results.

For the experiments, a sphere of $16 \mathrm{~mm}$ diameter and a cylinder of $20 \mathrm{~mm}$ diameter are used as exemplars for handled objects. An $8 \mathrm{~mm}$ indentation of both geometries 


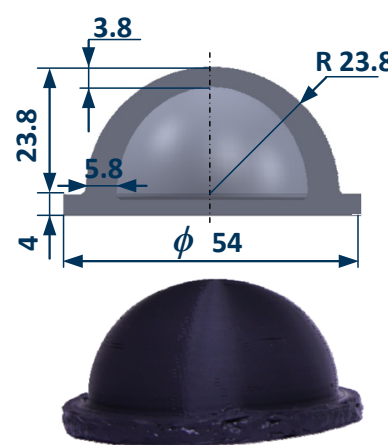

(a)

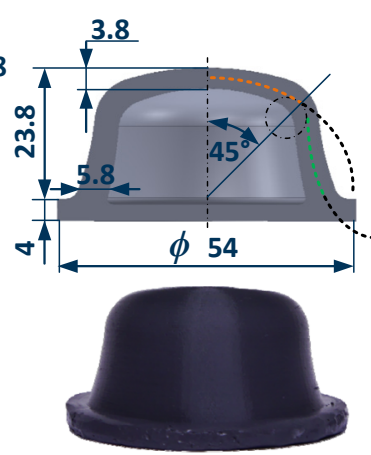

(b)

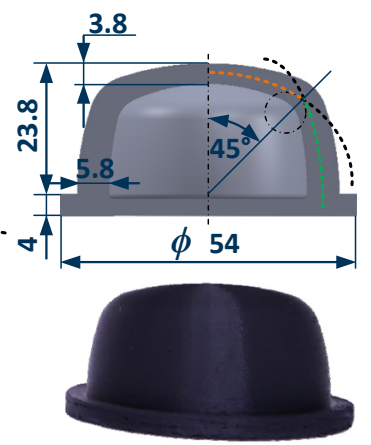

(c)

Figure 21: Above: cross section and geometric details of the three studied samples. Below: photo of the manufactured samples. Cross-sections: (a) semi-spherical, (b) cylindrical-convex, (c) cylindricalconcave.

into the MSE samples is accomplished in the experiments. The experimental setup is shown in Figure 22. The actuator (a) provides displacement in the vertical axis to the elements bonded to it. The handled object (b) is fixed to a load cell (c) to measure the reaction forces during deformation. The MSE geometries (d) are placed on a structure (e) to allow the deformation. After the deformation, the magnetic field is applied to the MSE material by moving the magnet (f) closer to the sample. Finally, the shape adaptation quality is measured in radial direction of the sample using a triangulation laser sensor (g).

In the tests, the MSE end-effector is firstly deformed by the gripped object, and the shape is recorded on the MSE material after the application of the magnetic field through the permanent magnet, see Figure 20. For the simulations, the surrounding geometry of the MSE end-effector just after the deformation without the application of the magnetic field is numerically calculated by using the hyperelastic material model. The results are shown in Figure 23. A reason for the difference between theoretical and experimental results lies on the simplification of the model, which does not consider the encapsulated air. For further work, a coupling between a hyperelastic material model and a magnetostatic analysis is pending in order to enable a change of the initial shear modulus on the hyperelastic model depending on the magnitude of magnetic field.

\section{Future work and conclusion}

Instead of a detailed summary, which usually follows at this point, this concluding section presents a specific aspect of future work with initial ideas. From the presented results it is exhibited that the concept for locomotion and manipulation systems promises a real application in future. Nevertheless, there are still missing features on 


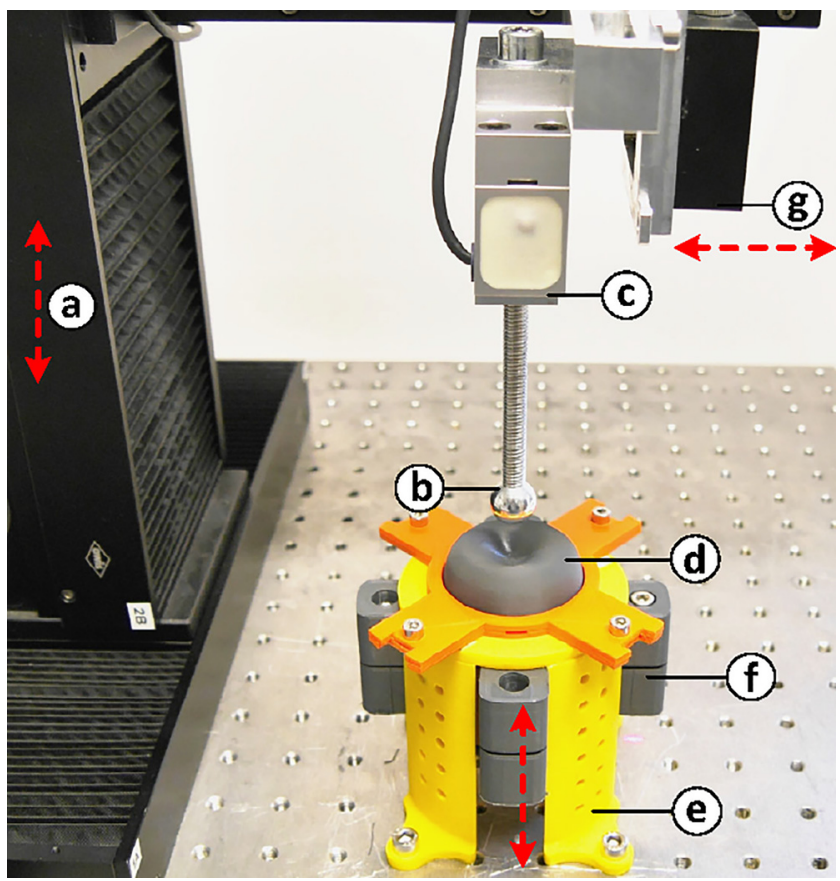

Figure 22: Experimental setup for shape adaptability and its components, a: displacement actuator, b: handled object, c: load cell, d: MSE sample, e: structure, f: encapsulated permanent magnet, $\mathrm{g}$ : triangulation laser sensor.

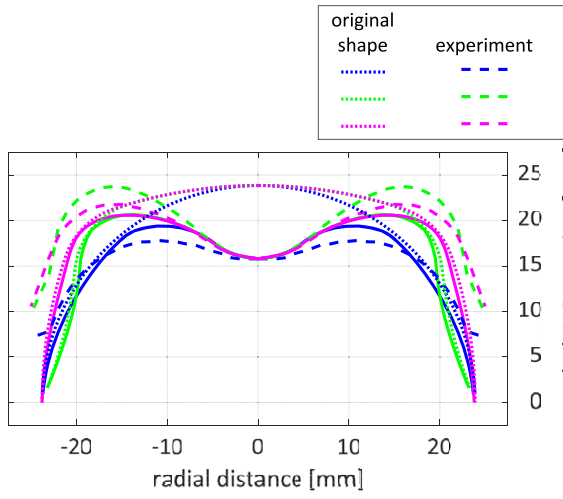

(a)

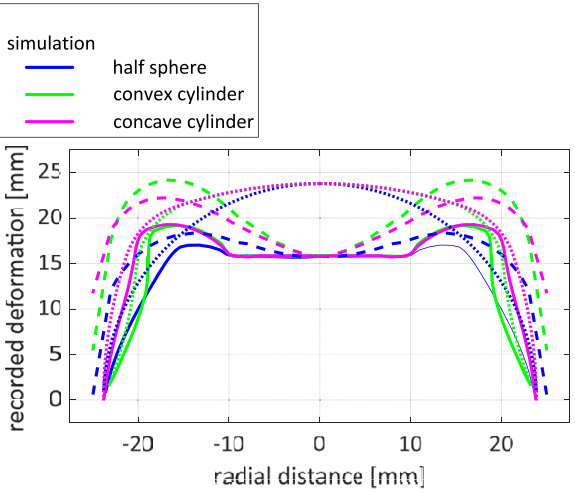

(b)

Figure 23: Experiments and simulations for shape adaptability of the three MSE end-effector prototypes for (a) sphere of $16 \mathrm{~mm}$ diameter and (b) cylinder of $20 \mathrm{~mm}$ diameter as gripped object.

these systems to achieve a technical implementation. One of the most needed attributes is a sensor integration on the introduced actuators, so integrated sensor-actuator systems can be achieved. Thus, this section describes a first concept for a sensory 

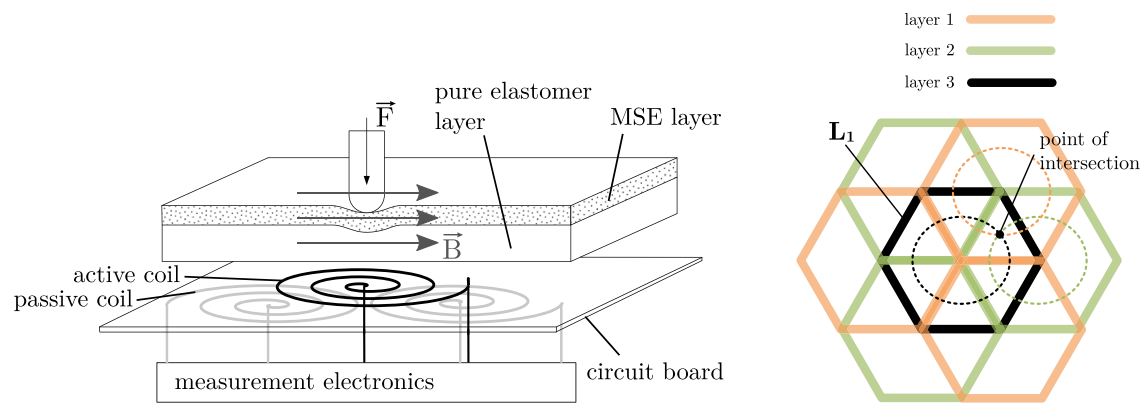

Figure 24: Tactile sensor concept (left), example of a planar coil array with seven hexagonal coils (right).

element which can be attached to the described prototypes. Additionally, a short summary of the results is given at the end of this chapter.

\subsection{Concept of an MSE based tactile sensor}

One aim of the future work is to combine sensitive effects and an active control of MSE by varying the magnetic field. The combination of MSE samples and inductors is investigated in [40, 41]. The following ideas and first design steps deal as well with an application incorporating both features.

The sensor concept shown in Figure 24 (left) is capable of sensing tactile information and controlling elasticity at once. In order to obtain information on a deformation, the sensor incorporates a circuit board with multiple planar spiral coils. The sensor consists of an elastic (MSE and pure elastomer) and a rigid (circuit board) part. The elastomer sample is made by a thin MSE layer and a pure elastomer base layer. The coils are connected to measurement electronics that is capable of a sequenced measurement of inductance. Therefore, every single inductance is time shifted part of an L-C-tank circuit driven by its eigenfrequency. Every deformation induces a change of reluctance near the spiral coil, thereby affecting the inductance. The measurements are performed with a high frequency alternating magnetic field $(\approx 2 \mathrm{MHz})$ at low amplitudes. Thus, an external induced magnetic field is superposed with the low magnitude field of the coils, controlling the elasticity of the MSE layer. The features of the sensor are a planar mapping and force measurement of an indentation as well as a tunable elasticity.

The example shown in Figure 24 (right) is used to visualize the principle for a planar mapping of a shifted indentation of constant force. This setup consists of three circuit board layers holding seven hexagonal coils in total. The change of inductance for a planar shifted indentation has a radial dependency to the centre of a spiral coil [42]. It is assumed, that for one coil all values of equal change of inductance can be fitted with an ellipse. It is necessary to determine the ellipses of one coil per layer that are close to the indentation. 


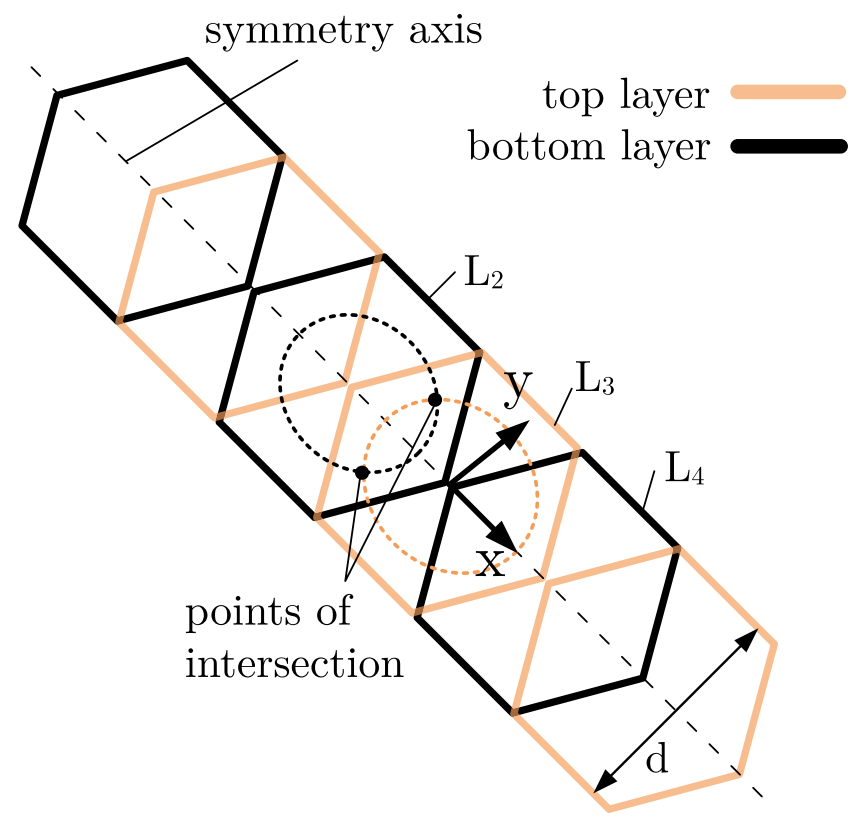

Figure 25: Realised array of coils for a linear tactile sensor.

Thus, one point of intersection can be found for every planar shifted indentation within the region of the coil $L_{1}$ (Figure 24, right). The coordinates of this point correspond to the planar force-applied position. For this sensor concept, it is required that there is still a positive change of inductance outside of the coil dimensions.

A first realised approach is the linear coil array shown in Figure 25. This circuit board holds multiple hexagonal coils arranged in a line. Due to its symmetry, the points of intersection have the same $x$-coordinate. The experimental setup consists of a three axis linear drive holding the indenter and a fixed tactile sensor with a layered composition as shown in Figure 24. For the given parameters listed in Table 1, there is a positive change of inductances measured outside of the coil dimensions, Figure 26. As a result, this setup is suitable for a linear positioning of an indentation.

Further information on the deformation can be obtained by measuring the inductance of a series connection of multiple coils. Next steps focus on using this data for evaluating the magnitude of the force.

\subsection{Compact summary of the results}

The use of MSE allows the realisation of actuator systems with a complex and adaptable mechanical behaviour, primarily resulting from the magnetically field-controlled particle-matrix interactions. The implementation of the beneficial properties of MSE in prototypes for locomotion and manipulation purposes is considered. The 
Table 1: Experimental parameters.

\begin{tabular}{ll}
\hline Parameters & Values \\
\hline Indenter shape & Ball \\
Indenter diameter & $5 \mathrm{~mm}$ \\
Coil diameter d & $23 \mathrm{~mm}$ \\
Coil turns & 30 \\
Indentation & $1 \mathrm{~mm}$ \\
MSE thickness & $2 \mathrm{~mm}$ \\
Pure elastomer thickness & $6 \mathrm{~mm}$ \\
Distance between layers & $0.5 \mathrm{~mm}$ \\
Capacitance & $120 \mathrm{pF}$ \\
Grid dimension & $2 \mathrm{~mm}$ \\
Measurement range $y$ & -26 to $26 \mathrm{~mm}$ \\
Measurement range $x$ & -6 to $6 \mathrm{~mm}$ \\
\hline
\end{tabular}

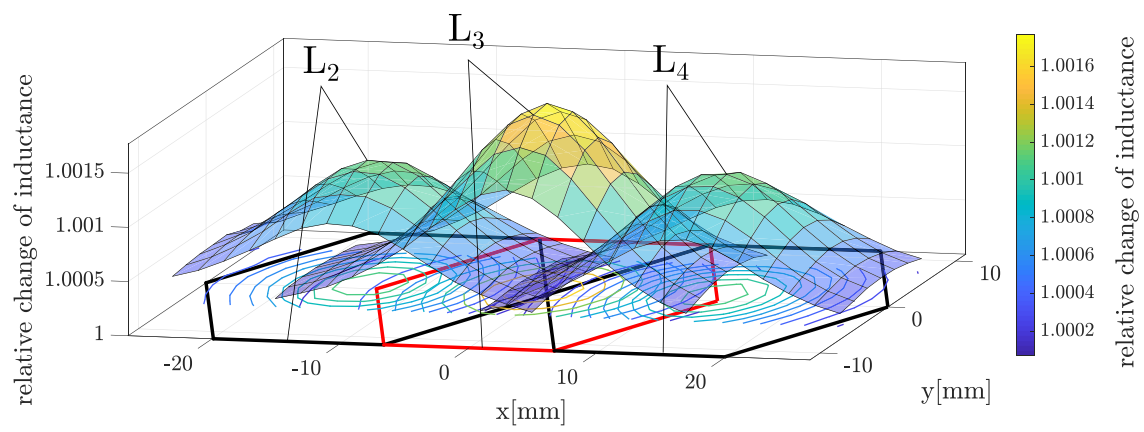

Figure 26: Experimental data of relative inductance change of a linear coil array.

investigations are formed by the steps: idea generation, model-based simulation, material synthesis, material characterization and building of prototypes. For technical solutions, two properties which are achievable through the systematic integration of the particles in the matrix and their interactions are important above all: the ability to create complex deformations by appropriate shaping of the objects due to the design of the magnetic fields and the realisation of a reversible variable, by magnetic fields controllable, mechanical compliance. The development of integrated sensor-actuator systems is defined as a new research subject with great potential.

Acknowledgement: This work was supported by Deutsche Forschungsgemeinschaft (DFG) within the project ZI540-17/1-3, in the framework of the priority program SPP 1681. We thank our partners in the SPP 1681 for the inspiring cooperation. Special thanks go to the teams of Prof. S. Odenbach (TU Dresden), Prof. M. Schilling (TU 
Braunschweig), Dr. R. Müller (IPHT Jena), Prof. S. Dutz (TU Ilmenau) and Prof. G. Monkmann (OTH Regensburg). Financial support by DFG under the project BE6553/1-1 within PAK 907 is also gratefully acknowledged. This work was carried out with the helpful support of numerous German and foreign scientists, who always showed a great interest in our work. We especially thank Prof. J. Steigenberger (Ilmenau), Prof. N.N. Bolotnik (Moscow) and Prof. V.A. Naletova (Moscow).

Author contribution: All the authors have accepted responsibility for the entire content of this submitted manuscript and approved submission.

Research funding: This work was supported by Deutsche Forschungsgemeinschaft (DFG) within the project ZI540-17/1-3 (SPP 1681) and BE6553/1-1 (PAK 907).

Conflict of interest statement: The authors declare no conflicts of interest regarding this article.

\section{References}

1. Becker TI, Raikher YuL, Stolbov OV, Böhm V, Zimmermann K. Dynamic properties of magnetosensitive elastomer cantilevers as adaptive sensor elements. Smart Mater Struct 2017;26:095035.

2. Böse H, Rabindranath R, Ehrlich J. Soft magnetorheological elastomers as new actuators for valves. J Intell Mater Syst Struct 2012;23:989-94.

3. Melenev PV, Raikher YL, Rusakov VV. Structure-mechanical model for plasto-elastic behavior of soft magnetic elastomers. Polym Sci 2010;52:430-5.

4. Odenbach S. Microstructure and rheology of magnetic hybrid materials. Arch Appl Mech 2016;86: 269-79.

5. Zimmermann K, Böhm V, Kaufhold T, Chavez Vega J, Becker T, Odenbach S, et al. Investigations and simulations on the mechanical behavior of magneto-sensitive elastomers in context with soft robotic gripper application. Int Sci J IFToMM Probl Mech 2016;65:13-26.

6. Volkova TI, Böhm V, Kaufhold T, Popp J, Becker F, Borin DYu, et al. Motion behaviour of magnetosensitive elastomers controlled by an external magnetic field for sensor applications. J Magn Magn Mater 2017;431:262-5.

7. Nadzharyan TA, Sorokin VV, Stepanov GV, Bogolyubov AN, Kramarenko EYu. A fractional calculus approach to modeling rheological behavior of soft magnetic elastomers. Polymer 2016;92: $179-88$.

8. Bica I. The influence of the magnetic field on the elastic properties of anisotropic magnetorheological elastomers. J Ind Eng Chem 2012;18:1666-9.

9. Tian TF, Li WH, Alici G. Study of magnetorheology and sensing capabilities of mr elastomers. J Phys Conf 2013;412:012037.

10. Zając P, Kaleta J, Lewandowski D, Gasperowicz A. Isotropic magnetorheological elastomers with thermoplastic matrices: structure, damping properties and testing. Smart Mater Struct Mar 2010; 19:045014.

11. Morgenstern AH, Calascione TM, Fischer NA, Lee TJ, Wentz JE, Nelson-Cheeseman BB. Thermoplastic magnetic elastomer for fused filament fabrication. AIMS Mater Sci 2019;6: matersci-06-03-363.

12. Prem N, Chavez Vega J, Böhm V, Sindersberger D, Monkman GJ, Zimmermann K. Properties of polydimethylsiloxane and magnetoactive polymers with electroconductive particles. Macromol Chem Phys 2018;219:1800222. 
13. Becker TI, Raikher YL, Stolbov OV, Borin D, Stepanov G, Böhm V, et al. Magnetic state of premagnetised magnetic hybrid elastomers with a complex filler. In: Proceedings of the 6th Colloquium of SPP 1681, Benediktbeuern, September 26-28, 2018; 2018:10-11 pp.

14. Becker TI, Zimmermann K, Borin DY, Stepanov GV, Storozhenko PA. Dynamic response of a sensor element made of magnetic hybrid elastomer with controllable properties. J Magn Magn Mater 2018;449:77-82.

15. Han Y, Mohla A, Huang X, Hong W, Faidley LE. Magnetostriction and field stiffening of magnetoactive elastomers. Int J Appl Mech 2015;7:1550001.

16. Saga N, Nakamura T. Elucidation of propulsive force of microrobot using magnetic fluid. J Appl Phys 2002;91:7003-5.

17. Saga N, Nakamura T. Development of a peristaltic crawling robot using magnetic fluid on the basis the locomotion mechanism of the earthworm. Smart Mater Struct 2004;13:566-9.

18. Kimura T, Umehara Y, Kimura F. Magnetic field responsive silicone elastomer loaded with short steel wires having orientation distribution. Soft Matter 2012;8:6206.

19. Zrinyi M, Barsi L, Büki A. Deformation of ferrogels induced by nonuniform magnetic fields. J Chem Phys 1996;104:566-9.

20. Zrinyi M, Barsi L, Szabo D, Kilian H-G. Direct observation of abrupt shape transition in ferrogels induced by nonuniform magnetic field. J Chem Phys 1997;106:5685-92.

21. Kashima S, Miyasaka F, Hirata K. Novel soft actuator using magnetorheological elastomer. IEEE Trans Magn 2012;48:1649-52.

22. Naletova VA, Zimmermann K, Zeidis I, Turkov VA, Kalmykov SA, Lukashevich MV. Dynamics of a prolate magnetizable elastic body in a cylindrical channel. In: 7th EUROMECH solid mechanics conference, Lisbon, Portugal, September 7-11, 2009: 2009:259-60 pp.

23. Zimmermann K, Zeidis I, Naletova VA, Kalmykov SA, Turkov VA. Modelling of a locomotion device based on deformable thin rod with magnetizable composite. In: 20th international conference on soft magnetic materials, Kos Island, Greece, September 18-22, 2011:1 p.

24. Zimmermann K, Zeidis I, Naletova VA, Turkov VA. Modelling of worm-like motion systems with magneto-elastic elements. Phys Status Solidi 2004;1:3706-9.

25. Zimmermann K, Zeidis I, Naletova VA, Turkov VA, Stepanov GV, Lukashevich MV. Undulation of magnetizable elastic body in magnetic field. In: Third Moscow international symposium on magnetism, Moscow, Russia, June 25-30; 2005; 2005:86-9 pp.

26. Phan KL, Mauritz A, Homburg FGA. A novel elastomer-based magnetoresistive accelerometer. Sensor Actuator Phys 2008;145-146:109-15.

27. Pettersson A, Davis S, Gray J0, Dodd TJ, Ohlsson T. Design of a magnetorheological robot gripper for handling of delicate food products with varying shapes. J Food Eng 2010;98:332-8.

28. Melenev P, Raikher Y, Stepanov G, Rusakov V, Polygalova L. Modeling of the field-induced plasticity of soft magnetic elastomers. J Intell Mater Syst Struct 2011;22:531-8.

29. Chavez Vega J, Kaufhold T, Schümann M, Böhm V, Zimmermann K, Odenbach S. Soft robotic gripping of sensible objects using field-induced plasticity of magneto-sensitive elastomers. In: 11th European magnetic sensors and actuators conference, Torino, 2016; 2016:259-60 pp.

30. Han Y, Hong W, Faidley L. Field-stiffening effect of magneto-rheological elastomers. Int J Solid Struct 2013;50:2281-8.

31. Chavez Vega J, Kaufhold T, Böhm V, Becker T, Zimmermann K, Martens M, et al. Field-induced plasticity of magneto-sensitive elastomers in context with soft robotic gripper applications. Proc Appl Math Mech 2017;17:23-6.

32. Chavez Vega J, Schorr P, Scharff M, Schale F, Böhm V, Zimmermann K. Towards magneto-sensitive elastomers based end-effectors for gripping application technologies. In: 2019 IEEE international conference on mechatronics (ICM), Ilmenau, March, 18-20, 2019; 2019:217-22 pp. 
33. Becker TI, Böhm V, Chavez Vega J, Odenbach S, Raikher Y, Zimmermann K. Magnetic-fieldcontrolled mechanical behavior of magneto-sensitive elastomers in applications for actuator and sensor systems. Arch Appl Mech 2019;89:133-52.

34. Condeelis J. Life at the leading edge: formation of cell protrusion. Annu Rev Cell Biol 1993;9: 414-40.

35. Bogolyubov NN, Mitropolski YA. Asymptotic methods in the theory of nonlinear oscillations. New York: Gordon and Breach Science; 1961.

36. Zimmermann K, Zeidis I, Bolotnik N, Pivovarov M. Dynamics of a two-module vibration-driven system moving along a rough horizontal plane. Multibody Syst Dyn 2009;22:199-219.

37. Stossel ThP. On the crawling of animal cells. Science 1993;260:1086-94.

38. Zentner L, Linß S. Compliant systems. New York: DeGruyter; 2019.

39. Zimmermann K, Chavez Vega J, Becker T, Witte H, Schilling C, Köhring S, et al. An approach to a form-adaptive compliant gripper element based on magneto-sensitive elastomers with a bioinspired sensorized surface. Int Sci J IFToMM Probl Mech 2019;75:23-38.

40. Alekhina Y, Makarova L, Rusakova T, Semisalova A, Perov N. Properties of magnetorheological elastomers in crossed ac and dc magnetic fields. J Sib Fed Univ Math Phys 2017;10:45-50.

41. Kawasetsu $\mathrm{T}$, Horii $\mathrm{T}$, Ishihara $\mathrm{H}$, Asada $\mathrm{M}$. Flexible tri-axis tactile sensor using spiral inductor and magnetorheological elastomer. IEEE Sensor J 2018;18:5834-41.

42. Kawasetsu T, Horii T, Ishihara H, Asada M. Size dependency in sensor response of a flexible tactile sensor based on inductance measurement. In: IEEE sensors; 2017:3 p. https://doi.org/10.1109/ icsens.2017.8233908. 